\title{
Pesquisa de Sangue Oculto nas Fezes e Correlação com Alterações nas Colonoscopias
}

\author{
Faecal Occult Blood Test and it's Correlation with Colonoscopic Results
}

\author{
FRANCISCO LUIS ALTENBURG'; ${ }^{1}$ MARIADELOURDES PESSOLE BIONDO-SIMÕES²; ALINE SANTIAGO³ $^{3}$
}

\author{
${ }^{1}$ Membro Titular da Sociedade Brasileira de Coloproctologia, Mestrando em Cirurgia na Universidade Católica do \\ Paraná, Curitiba, Paraná. Preceptor de Residência Médica em Cirurgia Geral e Coloproctologia do Hospital \\ Municipal São José e Hospital Regional Hans Dieter Schmidt, Joinville, Santa Catarina; ${ }^{2}$ Doutora em Técnicas \\ Operatórias e Cirurgia Experimental, Mestre em Morfologia, Professora Titular Da Pontifícia Universidade Católica do \\ Paraná, Professora Adjunta da Universidade Federal do Paraná; ${ }^{3}$ Residente de Coloproctolgia do Hospital Municipal \\ São José, Joinville, Santa Catarina.
}

\begin{abstract}
ALTENBURG FL; BIONDO-SIMÕES MLP; SANTIAGO A. Pesquisa de Sangue Oculto nas Fezes e Correlação com Alterações nas Colonoscopias. Rev bras Coloproct, 2007;27(3): 304-309.

RESUMO: Introdução: Diante do número significativo de casos de câncer colo-retal métodos de rastreamento tornam-se necessários. A pesquisa de sangue oculto (PSO) e tida como apropriada para grandes populações consideradas de baixo risco.. Objetivo: Determinar a incidência de lesões neoplásicas ou pré-neoplásicas em pacientes com PSO positivos ou negativos, e correlacionar com os achados da colonoscopia. Métodos: 67 pacientes foram submetidos à colonoscopia e PSO. As indicações para os exames foram: dor abdominal, alteração do hábito intestinal, e história familiar de câncer colo-retal. Resultados: 37 pacientes tinham PSO negativa e 30 PSO positiva. Vinte e duas colonoscopias foram normais. As colonoscopias revelaram : pólipos, retocolite ulcerativa, doença diverticular e um tumor. A sensibilidade foi de $46,7 \%$, especificidade de $59,1 \%$, valor preditivo positivo de $70 \%$ e negativo de $35,1 \%$. Considerando apenas as lesões malignas ou com potencial, a sensibilidade foi de 76,5\%, a especificidade de $66 \%$, o valor preditivo positivo de $43,3 \%$ e o negativo de $89,2 \%$. Conclusões: Há necessidade de rastreamento a partir dos 40 anos. A pesquisa de sangue oculto nas fezes, pelo seu baixo custo e caráter não invasivo, apesar da baixa sensibilidade e especificidade, é um bom método para rastreamento de populações consideradas de baixo risco.
\end{abstract}

Descritores: sangue oculto, neoplasia colorretais, colonoscopia, prevenção secundária, pólipos intestinais.

\section{INTRODUÇÃO}

O câncer colo-retal é o quinto câncer mais diagnosticado no Brasil, e no sudeste do país ocupa o segundo lugar sendo a quarta causa mais importante de mortes por câncer no país ${ }^{1}$.

É o quarto mais freqüente no mundo e o segundo nos países desenvolvidos. No Brasil, o Instituto Nacional do Câncer (INCA) relatou, no ano de 2006, incidência de câncer colo-retal de 12,36 novos casos para cada 100.000 homens, e de 14,73 para cada 100.000 mulheres. Este número foi diferente nas regiões e estados brasileiros. Mostrou-se baixo na região norte, especialmente no estado do Amapá, onde a incidência foi de 0,95 casos para cada 100.000 homens e 1,38 para cada 100.000 mulheres. Apareceu como o terceiro mais freqüente na região sul. O estado do Rio Grande do Sul foi o que apresentou a maior incidência brasileira com 27,68 novos casos para cada 100.000 homens e 28,07 para cada 100.000 mulheres $^{2}$.

Os sintomas do câncer colo-retal, na maioria dos pacientes, surgem quando a doença está avançada, demandando maiores custos de tratamento e altas taxas de morbidade e mortalidade. Considerando a crescente incidência desta doença, o diagnóstico precoce dela e mesmo de doenças predisponentes se torna im- 
portante. Programas de detecção são caros e limitantes para o sistema de saúde do Brasil.

A Sociedade Brasileira de Coloproctologia juntamente com o Instituto Nacional de Câncer, o Colégio Brasileiro dos Cirurgiões e outras sociedades médicas recomendam que indivíduos de baixo risco, a partir de 50 anos, realizem, anualmente, a pesquisa de sangue oculto nas fezes e a retossigmoidoscopia, repetindo-a a cada cinco anos. A partir dos 60 anos, está indicada a colonoscopia ou o enema opaco a cada 10 anos $^{1}$. A Sociedade Americana de Coloproctologia, no entanto, recomenda iniciar o rastreamento a partir dos 40 anos e a colonoscopia a partir dos $50 \operatorname{anos}^{3}$. A pesquisa de sangue oculto positiva, implica na realização de colonoscopia.

A população de maior risco inclui indivíduos maiores de 50 anos, com história pessoal ou familiar de pólipos e/ou câncer de intestino, retocolite ulcerativa, doença de Crohn e câncer de mama, ovário ou útero. Este grupo deve iniciar o rastreamento aos 40 anos, incluindo a colonoscopia ${ }^{1}$.

A pesquisa de sangue oculto (PSO) nas fezes surgiu como alternativa para o rastreamento do câncer colo-retal em pacientes sem fatores de risco. Este teste se sustenta no princípio de que os carcinomas do cólon sangram e que esta hemorragia oculta pode ser identificada pela PSO, que é um exame facilmente disponível. A pesquisa de sangue oculto consiste na identificação de hemoglobina nas fezes, podendo ser realizado pelos métodos: teste da o-tolidina (tradicional) e o teste imunológico, que detectam especificamente a hemoglobina humana ${ }^{4}$.

O teste da o-tolidina ou guaiaco foi desenvolvido por Van Deen, em 1864, e utilizado por Boas, em 1901, para pesquisa de sangramento gástrico. Passou a ser utilizado, em 1967, para monitoramento doméstico de sangue oculto nas fezes ${ }^{5}$. Baseia-se na atividade pseudoperoxidase que a porção da hemoglobina exerce, causando a oxidação de um composto fenólico (ácido alfa-guaiacônico) pela ação do peróxido de hidrogênio da solução de desenvolvimento, formando uma estrutura quinona. Esta reação química torna-se visível pelo aparecimento da cor azul ou azul esverdeada, dentro de 30 segundos, se sangue estiver presente nas fezes ${ }^{6}$.

No Brasil, muitos laboratórios utilizam o reativo de Meyer para o processo descrito acima. Esta mistura, quando adicionada de água oxigenada, adquire coloração avermelhada na presença de hemoglobina. As diferentes variações da coloração avermelhada são classificadas em positivas de uma a quatro cruzes.

Apesar da introdução da aglutinação de látex imunoquímico por Adams et al., em 1974, da imunodifusão radial simples por Songster et al., em 1980 e da hemaglutinação passiva reversa por Solto et al. em 1984, que levaram a métodos mais sensíveis para detectar baixas concentrações de hemoglobina humana nas fezes, o método permanece deficiente e sofre várias influências. A utilização de certos alimentos, drogas, vitaminas e outras substâncias antes e durante o período de coleta da amostra alteram o resultado podendo fornecer resultado falso positivo ou falso negativo, necessitando que o paciente faça dieta específica por 3 a 4 dias antes de realizar o teste ${ }^{5,6}$.

Estudos mostram que os resultados do teste são positivos somente em cerca de 50 a $65 \%$ dos pacientes com câncer colo-retal e em 25 a $40 \%$ em pacientes com pólipos ${ }^{5,7}$.

O teste imunológico ou teste de imunocromatografia de captura, desenvolvido em 1995, utiliza anticorpos monoclonais e policlonais para possibilitar a detecção de amostras com níveis tão baixos quanto $0,05 \mathrm{ul} / \mathrm{ml}$ em 5 minutos. Esta reação imunológica elimina a necessidade de dieta alimentar antes da coleta, assim como as interferências de hemoglobinas de outras espécies, realidades para os testes utilizando guáiaco ${ }^{6}$. A coleta da amostra não deve ser realizada durante ou dentro de três dias de um período menstrual, nem em paciente com queixa de hematoquezia ou com hematuria. Nestas situações os resultados do teste podem ser falso-positivos. Pacientes em uso de aspirina ou álcool devem suspender o uso uma semana antes da realização do exame ${ }^{5}$. Os testes imunológicos, por não necessitarem de dieta prévia e serem específicos para a hemoglobina humana, são citados na literatura como os de melhor sensibilidade $(75 \%)$ e especificidade $(40 \text { a } 50 \%)^{8}$.

Woo et al. empregando o teste imunoquímico para a pesquisa de sangue oculto, encontraram sensibilidade de $58,3 \%$, especificidade de $76,3 \%$ e valor preditivo positivo de $27,9 \%$, para o câncer colo-retal e ademonas ${ }^{9}$.

Levi et al. fizeram um estudo comparando a pesquisa feita com guáiaco e com a imunoquímica. A sensibilidade do guáiaco foi de $75 \%$ e o mesmo para a imunoquímica. A especificidade do teste feito com o guáiaco foi de $34 \%$ e a com a imunoquímica foi de $94 \%$. O valor preditivo positivo do guáiaco foi de $12 \%$ contra $60 \%$ com o teste feito pela imunoquímica ${ }^{10}$. 
Vê-se, portanto que existem discordâncias quanto ao poder de rastreamento destes testes. Além disso, segundo Morikawa et al. a sensibilidade não só é baixa como é diferente conforme a localização da lesão, quanto mais distal mais elevada e quanto mais proximal mais baixa ${ }^{11}$.

No nosso país, tanto o Ministério da Saúde quanto o Instituto Nacional do Câncer têm preconizado o rastreamento das populações com mais de 50 anos com o uso da pesquisa de sangue oculto, ficando a busca endoscópica limitada aos pacientes com sangue positi$\mathrm{vo}^{2}$. A busca ativa endoscópica, preconizada acima dos 50 anos, por ter custo mais elevado, acaba sendo privilégio de minorias.

\section{OBJETIVO}

Avaliar a incidência de lesões neoplásicas e pré neoplásicas em pacientes com PSO positiva ou negativa, e em pacientes sintomáticos submetidos à colonoscopia.

\section{MÉTODOS}

Trata-se de um estudo transversal no qual se analisam os resultados de colonoscopia e de pesquisa de sangue oculto em pacientes sintomáticos. Foram incluídos no estudo 67 pacientes com indicação de colonoscopia, de janeiro de 2006 a abril de 2007. Eram 43 mulheres e 24 homens com média de idade de 52 e 53 anos respectivamente. $O$ paciente mais jovem tinha 23 anos e o mais velho 81 anos. Nenhum paciente tinha realizado este exame anteriormente e todos foram realizados por um único examinador. Foram analisados os pacientes, as indicações clínicas dos exames, o resultados das colonoscopias e a relação dos achados de lesões neoplásicas e pré neoplásicas com a PSO.

Os resultados, forneceram a sensibilidade, a especificidade, o valor preditivo positivo e o valor preditivo negativo, com intervalo de confiança de $95 \%$.

\section{RESULTADOS}

A pesquisa de sangue oculto revelou-se negativa em 37 pacientes e positiva em 30 deles.

As indicações para as colonoscopias foram: dor abdominal, alterações do hábito intestinal (diarréia ou constipação), familiares de primeiro grau com antecedentes de câncer colo-retal, história de sangramento anterior ou a combinação destes sintomas. Todos os pacientes realizaram previamente a pesquisa para sangue oculto pelo método imunológico.

Todas as colonoscopias alcançaram o ceco(23) ou o íleo(44)? Vinte e duas colonoscopias foram normais $(31,83 \%)$ e 45 colonoscopias positivas $(68,17 \%)$. Os diagnósticos foram: pólipos, doença diverticular, pólipos associados à doença diverticular, retocolite ulcerativa, tumores e outras. A relação de pólipos, retocolite e tumores com a PSO positiva ou negativa está listada na tabela 1.

Foram encontrados pólipos em 27 pacientes $(40,30 \%)$. Onze deles em pacientes com PSO positiva $(59,26 \%)$ e $16(40,74 \%)$ em pacientes com PSO negativa.. Além de pólipos foi encontrado um tumor( adenocarcinoma) de ceco e quatro pacientes com retocolite ulcerativa.

A sensibilidade da PSO neste estudo foi de $46,7 \%$ (Intervalo de confiança de $32,1 \%$ a $61,2 \%$ com IC de $95 \%$ ), a especificidade de $59,1 \%$ ( Intervalo de confiança de $38,5 \%$ a 79,6\% com IC de $95 \%$ ), a acurácia de $50,7 \%$ (Intervalo de confiança de $38,8 \%$ a $62,7 \%$ com IC de $95 \%$ ), valor preditivo positivo de $70 \%$ (Intervalo de confiança de 53,6\% a 86,4\% com IC de $95 \%$ ) e valor preditivo negativo de $35,1 \%$ (Intervalo de confiança de $19,8 \%$ a $50,5 \%$ com IC de $95 \%$ ) (Tabela 2).

Das 27 colonoscopias com polipectomia, 12 revelaram-se no exame anátomo-patológico como adenomas tubulares ou túbulo-vilosos, e as 15 restantes como pólipos inflamatórios ou hiperplásticos. Houve ainda quatro retocolites ulcerativas e um tumor.

Tabela 1 - Relação dos resultados das colonoscopias envolvendo lesões e a relação com a PSO positiva ou negativa.

\begin{tabular}{lccccc}
\hline & Pólipos & RCU & Tumor & LSPS* & Total \\
\hline PSO positiva $(\mathrm{n}=30)$ & 11 & 3 & 1 & 15 & 30 \\
PSO negativa (n=37) & 16 & 1 & 0 & 20 & 37 \\
Total & 27 & 4 & 1 & 35 & 67 \\
\hline
\end{tabular}

* Lesões sem potencial para sangramento. 
Considerando-se colonoscopias positivas as que revelaram adenoma e/ou adenocarcinoma e retocolite ulcerativas, a relação com a PSO positiva, a sensibilidade se eleva para 76,5\% (IC 95\%, 56,3 a 96,6), a especificidade para 66\% (IC 95\%, 52,9 a 79,1), o valor preditivo positivo para $43,3 \%$ (IC 95\%, 25,6\% a 61,1\%) e valor preditivo negativo de $89,2 \%$ (IC 95\%, 79,2 a 99,2) (Tabela 3).

\section{DISCUSSÃO}

O câncer colo-retal tem mostrado uma curva ascendente de incidência, nos países desenvolvidos. No Brasil este perfil se repete, basta observar que enquanto na região norte ocupa o quinto lugar nas causas de mortalidade, na região sudeste e na sul ocupa respectivamente a segunda e a terceira colocações ${ }^{2}$.

Quase a totalidade dos doentes busca atendimento quando já apresenta sintomas. Sabe-se que esta classe de tumores tem desenvolvimento silencioso, com sintomas apenas nas fases avançadas da doença, quando o tratamento radical nem sempre é possível, acarretando altas taxas de morbi-mortalidade.

Estudos realizados, em países desenvolvidos têm mostrado que o rastreamento de tumores, permite

Tabela 2 - Relação das colonoscopias positivas (lesões com potencial para sangramento) com a pesquisa de sangue oculto.

\begin{tabular}{lccc}
\hline & \multicolumn{3}{c}{ Colonoscopia } \\
\cline { 2 - 4 } & Positiva & Negativa & Total \\
\hline PSO positiva & 21 & 9 & 30 \\
PSO negativa & 24 & 13 & 37 \\
Total & 45 & 22 & 67 \\
\hline
\end{tabular}

Tabela 3 - Relação das colonoscopias com lesões neoplásicas elou com potencial para malignização e a PSO positiva ou negativa

\begin{tabular}{lccc}
\hline & \multicolumn{3}{c}{ Colonoscopia } \\
\cline { 2 - 4 } & Positiva & Negativa & Total \\
\hline PSO positiva & 13 & 17 & 30 \\
PSO negativa & 4 & 33 & 37 \\
Total & 17 & 50 & 67 \\
\hline
\end{tabular}

a detecção precoce deles e a diminuição da taxa de mortalidade. Assim nos Estados Unidos a redução da mortalidade foi de 14,5 para 9,1 para 100.000 habitantes ${ }^{1}$.

Estudos recentes têm utilizado a pesquisa de sangue oculto nas fezes como forma de screening e acompanhamento de pacientes sem fatores de risco para câncer colo-retal ${ }^{3,7,9}$. Os fatos demonstram que o exame anual de pesquisa de sangue oculto nas fezes pode diminuir em até $16 \%$ a mortalidade por câncer colo-retal $^{12}$.

Apesar do exame com o guáiaco sofrer influência de fatores como a dieta e o uso de medicações, permanece como um método acessível, barato, não invasivo e sem diferenças significativas em relação à especificidade, quando comparado ao teste imunológico. As alterações de sensibilidade do método são devido ao aumento do número de falsos positivos ${ }^{12}$. Estes falsos positivos implicam em exames dispendiosos ao sistema de saúde, porém não diminuem as possibilidades do diagnóstico precoce de câncer (o que acontece com falsos negativos), porém o custo não é proibitivo e deve ser estimulado como exame de rastreamento. Neste estudo a pesquisa de sangue oculto foi realizada pelo método imunoquímico. Portanto não sofrendo influência do tipo de dieta.

Todos os 17 pacientes com lesões malignas ou com potencial para malignidade, deste estudo, apresentavam idade acima dos 40 anos. Dois deles abaixo dos 50 anos. Estes dados reforçam as orientações da literatura para que o rastreamento se faça a partir dos $40 \operatorname{anos}^{1,3}$.

No grupo de pacientes com PSO positiva, apenas 7 pacientes eram assintomáticos, sendo que $72 \%$ destes pacientes apresentaram alterações nas colonoscopias. Quando avaliados os pacientes sintomáticos, constatou-se que $73 \%$ apresentavam alterações nos exames de colonoscopia e a queixa mais prevalente foi a dor ou o desconforto abdominal.

Os pacientes com PSO negativa com colonoscopia normal foram 33\%. A presença de pólipos representou o maior número de alterações encontradas nos exames(14 pacientes). A doença diverticular também foi representativa com 11 pacientes, ou seja $33 \%$ das alterações encontradas.

Os pacientes que apresentaram pólipos foram re-avaliados em busca de queixas, e constatou-se que do grupo de pacientes com PSO positiva, apenas um era assintomático, dois tinham antecedentes familiares 
Rev bras Coloproct Julho/Setembro, 2007 de câncer e os outros queixas de sangramento baixo, dor abdominal e/ou anemia. Os pacientes com pólipos, com PSO negativa tiveram como principal queixa a dor abdominal, e dois pacientes tinham antecedentes familiares de câncer colo-retal.

Existem vários meios de se fazer o rastreamento do câncer colo-retal e das doenças predisponentes. O enema opaco pode ser utilizado. É descrito com sensibilidade de 50 a $75 \%$ e especificidade de 80 a $85 \%$. Entretanto, a sensibilidade diminui sensivelmente quando as lesões são menores do que um centímetro $^{13}$. A tomografia computadorizada colonográfica tem sensibilidade de $90 \%$, porém também para esta modalidade de exame existe a dificuldade para a visualização de lesões menores do que um centímetro $^{14}$

A literatura mostra a pesquisa de sangue oculto com sensibilidade que varia entre 45 e $75 \%$, especificidade de 35 a $94 \%$ e valores preditivos positivos entre 28 e $60 \%{ }^{9-11}$. Neste estudo observou-se sensibilidade de $46,7 \%$, especificidade de $59,1 \%$, valor preditivo positivo de $70 \%$ e negativo de $35,1 \%$. Se entretanto, se considerar apenas as lesões neoplásicas ou com potencialidade para tal a sensibilidade atinge $76,5 \%$. Estes dados demonstram que é possível identificar verdadeiros positivos em 76,5\% dos examinados, e que a chance de um paciente com PSO positiva apresentar algum tipo de lesão com ou sem potencial para malignidade chega a 70\%. Estes dados justificam seu uso em populações de baixo risco.

Embora a colonoscopia seja o exame padrãoouro para o diagnóstico, considerando o baixo custo da pesquisa de sangue oculto nas fezes e o fato do exame não ser invasivo, este método pode ser tido como bom para o rastreamento do câncer colo-retal ${ }^{14,15}$.

O uso da PSO em indicações absolutas da colonoscopia é desnecessário, como por exemplo em pacientes com sangramento intermitente nos quais as doenças orificiais estejam ausentes, ou pacientes com antecedentes familiares de primeiro grau com câncer colo-retal, porém a PSO pode ser solicitada como exame preventivo a partir de 40 anos em pacientes assintomáticos e sem risco para o câncer colo-retal, ou mesmo em populações aonde o exame da colonoscopia é inviável.

\section{CONCLUSÃO}

A pesquisa de sangue oculto nas fezes, pelo seu baixo custo e caráter não invasivo, apesar da baixa sensibilidade e especificidade, é um bom método para rastreamento de populações consideradas de baixo risco.

\begin{abstract}
Methods of screening of colorectal cancer are necessaries, because the increasing number of new cases. The faecal occult test(fobt) is considered good for populations with low risk. The goal of this paper is to determine the incidence of neoplasic or pre neoplasic lesions in patients with fobt positive or negatives and correlate with the results of the colonoscopies. Sixtie seven patients were submited to colonocopies and fobt.the main indications were abddominal pain, familial history of cancer and modification of bowel habits. Thirty seven patients had negatives fobt and thirty positives.twenty two colonoscopies were normal. Others findings were polyps, ulcerative colitis, diverticular disease and one tumor. Sensitivity was $46.7 \%$ and specificity $59.1 \%$. Positive predictive values were $70 \%$ and negative $35.1 \%$. Considering only malignant or pre malignant lesions the sensitivity was $76.5 \%$ and specificity $66 \%$, positive predictive values were $43.3 \%$ and negative $89.2 \%$. In conclusion the faecal occult blood test for his low cost and non invasive is a good method of screening after forty years of age.
\end{abstract}

Key words: occult blood, intestinal neoplasm, intestinal polyps, secondary prevention, colonoscopy.

\section{REFERÊNCIAS}

1. Fang CB. Rastreamento do câncer colorretal. Ver Assoc Med. Bras. 2002; 48(4):286-6.

2. www.inca.gov.br. Câncer colo-retal. Acesso em 24 mai 2007.

3. American Cancer Society. Cancer facts and figures, 1999. Atlanta: American Cancer Society, 1999.

4. Novaes de Almeida FF, Araujo SEA, Santos FPS, Franco CJCS, Santos VR, Nahas SC, Harb-Gama A. Colorectal cancer screening. Rev Hosp Clin. 2000; 55(1):35-42.
5. http://analitic.com.br/feca_cult.php.

6. Moayyedi P; Achkar E. Does fecal occult blood testing really reduce mortality? A reanalysis of systematic review data. Am J Gastroenterol. 2006; 1001(2):380-4.

7. Screening for colorectal cancer using the faecal occult blood test, Hemoccult. Cochrane Database Syst Rev. 2007 Jan 24;(1):CD001216

8. Levi Z, Rozen P, Hazazi R, Waked A, Maoz E, Birkenfeld S, Niv Y. Can quantification of faecal occult blood predetermine the need for colonoscopy in patients at risk for non-syndromic 
familial colorectal cancer? Aliment Pharmacol Ther. 2006;124(10):1475-82.

9. Woo HY, Mok RS, Park YN, Park DI, Sung IK, Sohn CI, Park H. A prospective study of a new immunochemical fecal occult test in Korean patients referred for colonoscopy. Clin Biochem. 2005; 38(4):395-9.

10. Levi Z, Hazazi R, Rozen P, Vilkin A, Waked A, Niv Y. A quantitative immunochemical faecal occult test is more efficient for detecting significant colorectal neoplasia than a sensitive guaiac test. Aliment Pharmacol Ther. 2006; 23(9):1359-64.

11. Morikawa T, Kato J, Yamaji Y, Wada R, Mitsushima T, Shiratori Y. A comparison of the immunochemical fecal occult blood test and total colonoscopy in the asymptomatic population. Gastroenterology. 2005; 129(2):422-8.

12. Anderson WF; Guyton KZ; Hiatt RA; Vernon SW; Levin B; Hawk E. Colorectal cancer screening for persons at average risk. J Natl Cancer Inst. 2002;94(15):1126-33.

13. Winawer SJ, Stewart ET, Zauber AG, et al: A comparison of colonoscopy and double-contrast barium enema for surveillance after polypectomy: National Polyp Study Work Group. N Engl J Med 2000; 342:1766.

14. Rex DK, Lehman GA, Hawes RH, et al: Colorectal cancer prevention 2000: Screening recommendations of the American College of Gastroengeroloy. Am J Gastroenterol 2000; 95:868.

15. Ciatto S; Martinelli F; Castiglione G; Mantellini P; Rubeca T; Grazzini G; Bonanomi AG; Confortini M; Zappa M. Association of FOBT-assessed faecal $\mathrm{Hb}$ content with colonic lesions detected in the Florence screening programme. Br J Cancer. 2007; 96(2):218-21.

16. Müller AD, Sonnenberg A. Protection by endoscopy against death from colorectal cancer. A case-control study among veterans. Arch Intern Med 1995; 155:1741-8.

\section{Endereço para correspondência:}

FRANCISCO LUIS ALTENBURG

Rua XV de Novembro 2119, Casa 14, Joinville, Santa Catarina, CEP 89201620.

E-mail: progastro@terra.com.br

Fax: 047 3026-2133 